\title{
Clinical utility of cognitive screening instruments in dementia patients of neurology OPD (Memory Clinic) in Eastern Uttar Pradesh, India
}

\author{
S. Kant ${ }^{1, *}$, Karan Poddar $^{2}$, M. Kamle ${ }^{3}$, C. Patil ${ }^{4}$ \\ Consultant, ${ }^{1,2}$ Neurology Centre, Varanasi, Uttar Pradesh, ${ }^{3}$ Kamle Clinic, Vikroli, Mumbai, Maharashtra, ${ }^{4}$ Vardhman Clinic, \\ Thane, Maharashtra, India
}

*Corresponding Author:

Email: drskpoddar@gmail.com

\begin{abstract}
Introduction: Dementia is a major public health challenge that is becoming more common with growing age. It involves progressive and often remorseless decline in cognition, functions, behavior and care needs. The Assessment of dementia greatly relies on collateral as well as patient-derived information. The use of various cognitive tests assumes great importance during the process of diagnostic investigation. In this research we attempt to assess the practical usage of various cognitive scales.

Materials and Methods: In this study, we studied the data of 233 patients in the neurology OPD. The following cognitive scales like Montreal Cognitive Assessment (MoCA), Addenbrooke's Cognitive Examination (ACE), Hachinski Ischemic Score (HIS), and Everyday Abilities Scale for India (EASI) were performed on the above selected data of patient.

Results: 228 (97.85) \% of patients were found to have cognitive impairment when screened with MoCA. However, 230 (98.7\%) of patients screened with EASI had a disability score. The patients with a HIS score of more than 7 was (64) $27.9 \%$. Whereas, ACE indicated all patients had score below 83 (100\%). The sensitivity of MoCA, ACE, and EASI score was $72.69 \%$ and $72.29 \%$, $71.74 \%$ respectively with a specificity of 50 and $100,0.0 \%$ respectively for the above scores, whereas the sensitivity and specificity of HIS was $49.50 \%$ and 10.61 .

Conclusion: MoCA, ACE, and EASI tools have good capability to detect most patients with cognitive impairments. They are sensitive to predict probable cases of dementia, therefore can be used as an efficient diagnostic tool in quick screening of cognitive impairment.
\end{abstract}

Keywords: Montreal Cognitive Assessment (MoCA), Addenbrooke's Cognitive Examination (ACE), Hachinski Ischemic Score (HIS), Everyday Abilities Scale for India (EASI), and dementia.

\section{Introduction}

The worldwide prevalence of dementia is forecasted to double every 20 years, increasing from 24 million in 2001 to 40 million in 2020 and 80 million in $2040 .{ }^{1}$ In specific to India, it is predicted an estimate of 3.7 million people has dementia (2.1 million women and 1.5 million men). However, the prevalence of dementia would increase steadily with age. ${ }^{2}$ Most of the studies revealed that, the incidence and prevalence of dementia are strongly dependent on the age. With global aging of population, the prevalence of dementia also gradually rises and is projected to continue to do so far much of the present century. ${ }^{3}$

Most of literatures emphasized that, importance of early case identification, wherein primary care physicians have a key role, prior to onwards referral to dedicated dementia services, in the hope of narrowing or closing the diagnosis gap i.e. too few peoples being diagnosed with dementia or diagnosed early enough ${ }^{4}$. It's correct clinical identification and early diagnosis is a paramount importance.

The Linda Nazarko at el emphasized that the physicians have the very poor record of diagnosing people with dementia. ${ }^{5}$ Currently, approximate $75 \%$ of the patients with moderate to severe dementia are not recognized by primary care clinicians as despite of having cognitive impairment. However, David R at el claimed that, $97 \%$ of patients with mild cognitive impairment were not recognized as being in an early phase of a dementia syndrome. ${ }^{6}$

Early diagnosis of cognitive impairments would surely offer the best possible chances for their effective treatment. There have been many scales are devised in the field of dementia. The main purpose of the available screening tests is to enhance the precision of a decision by reducing subjectivity and increasing objectivity. Also, the reason behind early diagnosis is that, only third of patient with dementia received a diagnosis of dementia at any time of illness and aimed to formulate a plan to improve diagnostic rates For example using a cognitive screening test score test to screen for the underlying dementia, to distinguish impairment due to the dementia from normal age related cognitive change or to monitor the effects of treatment of dementia in a clinic or controlled Trial. ${ }^{6}$

Population studies of ageing and cognition suggest that impairment in multiple cognitive domains is observable several years before a clinical diagnosis of Alzheimer's disease is made. ${ }^{7}$ This perceived cognitive dysfunction is not qualitatively diverse than that seen in normal ageing, which suggestive of continuity rather than discontinuity in the shift from normal ageing to pre-clinical dementia. ${ }^{8}$ Global cognitive deterioration, affecting memory and other aspects of cognitive functioning (verbal ability, visuo-spatial skills, 
attention, and perceptual speed), is almost always a presenting symptom. ${ }^{8}$

There is considerable overlapping cognitive performance between normal ageing and this stable phase, and little evidence exists as yet that these changes are detectable in clinical encounters. A person with symptoms of Alzheimer's disease is about 30\% more likely to display the clinical features of dementia if they have coexisting symptoms of vascular disease. ${ }^{9}$

The progression of mild cognitive deficits can be delayed by using routine cognitive screening, which would help in early diagnosis and therefore early intervention..$^{10}$ There is ample evidence supporting the view that screening can improve case identification ${ }^{11}$. Besides, the routine cognitive screening not only facilitates early diagnosis and better treatment, but it also supports public health and fosters the research. Therefore, a good screening test plays a crucial role in planning for management of the disease.

The aim of this study was determine the prevalence of dementia at the out-patient department of (memory clinic) neurology (memory clinic) in Uttar Pradesh, to study the socio-demographic and illness related variables and to correlate various Scales. The assessment tools used were the Montreal Cognitive Assessment (MoCA), Addenbrooke's Cognitive Examination (ACE), Hachinski Ischemic Score (HIS) and Everyday Abilities Scale for India score (EASI).

\section{Materials and Methods}

In this study medical record of total 233 patients were studied. All 233 patients who had consulted at the neurology out-patient department during a period of one year from Jan-2016 to Jan-2017 \& who received a diagnosis of dementia as per the doctor's judgment underwent the detailed assessment tests, were screened $\&$ analyzed.

The MoCA is a cognitive screening instrument developed to detect MCI and dementia. It is a simple ten minute paper and pencil test to assess the various cognitive domains; it has seven sub scores; memory (5 points for delayed recall), language (3 points), visuospatial/ executive functions (5 points), abstraction ( 2 points), attention ( 6 points), naming ( 3 points)and orientation ( 6 points). Its validity has been established to detect $\mathrm{MCI}$ in patients with $\mathrm{AD}$ and other pathologies in cognitively impaired patients who scored in the normal range on the Mini-Mental State Examination (MMSE). ${ }^{12}$

The total possible score for MoCA screening instrument is 30 points with a score of 26 or more considered as a normal. ${ }^{12}$

The Addenbrooke's Cognitive Examination (ACE) and its revised version (ACE-R) are theoretically motivated revisions of the Mini-Mental State Examination (MMSE).

$\mathrm{ACE}$ and its subsequent iterations, ACE-R and ACE-III, have proved easy to use, acceptable to patients, and have shown excellent diagnostic utility in identifying dementia and cognitive impairment in a variety of clinical situations. ${ }^{13,14}$ The ACE-R takes between 12 and $20 \mathrm{~min}$ (average 16) to administer and score in a clinical setting. It comprises 5 sub-scores, each one representing one cognitive domain: attention/orientation (18 points), memory (26 points), fluency (14 points), language (26 points) and visuospatial (16 points). ACE-R maximum score is 100 , collectively by the addition of the all domains. ${ }^{15}$

It is scored out of 100 , with a higher score denoting better cognitive function. At the recommended cut-off scores of 88 and 83 , the ACE was reported to have good sensitivity and specificity for identifying dementia (0.93 and $0.71 ; 0.82$ and 0.96 , respectively). The ACE also incorporated the MMSE, such that this score (out of 30) might also be generated. ${ }^{16}$

Functional Ability Scale - Everyday Abilities Scale for India (EASI) - A new functional ability scale, in the form of an informant questionnaire, was developed to assist in dementia screening of this largely illiterate, rural, older Indian cohort. Functioning for daily living of the subjects was assessed in the presence of the primary caregiver with the help of EASI and subjects with EASI score of $>3$ were classified as functionally impaired. ${ }^{17}$

The Hachinski Ischemic Scale (HIS) is a tool widely used to identify a likely vascular component once a dementia diagnosis has been established. It is not itself a validated diagnostic tool. A score greater than seven suggests vascular involvement. ${ }^{18}$

\section{Statistical analysis}

The data was entered in the MS Excel software after completion for further processing. The qualitative data was presented in the form of number and percentage. The significant association of dementia with socio-demographic, behavioral and co-morbid variables was tested by the chi square test. The statistical calculation was performed using the Statistical Package of Social Sciences (SPSS), version 16.0 and Statistical analysis system (SAS) version 9.2.

\section{Results}

The study cohort consisted of 233 patients of which $189(81.1 \%)$ were men, $44(18.9 \%)$ were women. The mean age of the group at presentation was $63.45 \pm$ 11.59 years. The percentage of illiterate population was $10.7 \%$. Addiction was found in $119(50.9 \%)$ patients. (Table 1).

In this study $97.85 \%$ of patients were found to have cognitive impairment when screened with MoCA scale. Only 6 patients had a MoCA score of 26 or above. $230(98.7 \%)$ of patients screened with EASI had a disability score of EASI score of > 3. 64 (27.9\%) of patients has HIS score of more than 7. ACE validated for dementia evaluation, with a cut-off score of 83 
points showed all patients below 83 . The highest score in this category was 77 (Table 2).

This data emphasized that the study population showed abnormal values in all screening scales. 228 (97.85 \%) were cognitively impaired with MoCA, while all $233(100 \%)$ were impaired with ACE. Correlations matrix between age with the various scores like MoCA, EASI, HIS, and ACE was studied. Age is negatively correlated with MoCA score and ACE score and both are highly statistically significant at $0.01 \%$ $(\mathrm{P}<0.001)$. But EASI and HIS are not statistically significant at $5 \%$ level. The data showed a significant correlation of age with MoCA and ACE Score (Table 3).

However, Table 4 showed that, the comparison between males and female scores in MoCA, EASI, HIS, and ACE. The data showed that MoCA, EASI, and ACE are statistically significant. In MoCA and ACE are statistically highly significant at $0.01 \%$ level $(\mathrm{P}<0.001)$ and EASI is statistically significant at $5 \%$ level $(\mathrm{P}<0.05)$. Only HIS is not statistically significant at 5\% level $(\mathrm{P}>0.05)$ (Table 4$)$.

Table 5 showed that, the correlations matrix education with the various scores. The data showed a significant correlation of education with MoCA, EASI, and the ACE scores. However there is no significant association was found about type of diet and education with the HIS scores.

As Table 6 showed that, the sensitivity of MoCA, ACE, EASI score was $72.69 \%, 72.29 \%$, and $71.74 \%$ respectively, with a specificity of 50,100 , and $0.0 \%$ respectively for the above scores, whereas the sensitivity and specificity of HIS was $49.50 \%$ and $10.61 \%$. The sensitivity of MoCA and ACE, EASI Score were found to be extremely high which is indicative of the fact that these tools have a good capability to detect most patients with a morbid condition, i.e., it is sensitive enough to predict probable cases of dementia as compared to HIS.

\section{Discussion}

In an effort to reduce the burden, researchers have been focusing on precise screening procedures that might detect the possible cases in advance or prevent or slow the rate of progression of this disease, as early diagnosis of dementia is essential to any treatment effort ${ }^{19}$. In this study, we evaluate the practical use of cognitive scales for the domain of cognitive functioning, behavior, quality of life, depressive behavior in dementia, and overall dementia severity.

Research on dementia and other chronic diseases in developing countries have lagged behind research taking place in the developed countries. Reasons are multiple and complex but include overall limitations of resources, and lack of appropriate assessment tools. With respect to dementia, there has been a lack of adequate screening instruments for the rapid and accurate identification of potentially demented individuals, particularly in populations that are poorly educated and vastly diverse in language and culture. Such instruments would also be of value in the assessment of poorly educated patients and research subjects anywhere. ${ }^{20}$

The present study was conducted in patients with dementia; who visited neurology OPD. The mean age of the group at presentation was $63.45 \pm 11.59$ years. Correlations matrix between age with the various scores like MoCA, EASI, HIS, and ACE was done where age is negatively correlated with MoCA score and ACE score and both are statistically significant at $0.01 \%$ $(\mathrm{P}<0.001)$. This shows that MoCA score and ACE reduces with advancing age.

More elderly people living by themselves and even mild cognitive impairment may prove significantly disturbing for the patient in his day to day life, making them seek medical help. Thus the mean age at presentation may decrease in the next few years. ${ }^{21,22}$

In this study, there was more number of male patients than female patients. The reason for increased number of male patients in our study could perhaps be explained in the socio-cultural context wherein elderly males may continue to be actively engaged in activities, which may make cognitive deficits more apparent in them. Elderly females on the other hand may be involved in relatively simple roles within the household, while the more complex tasks like cooking or buying of grocery may be delegated to the younger generation.

In the present study, $97.85 \%$ of patients were found to have dementia when screened with MoCA scale. The higher incidence could be because most patients were visiting neurology OPD having some features of cognitive impairment. In this study, the patients with advance age, low education and female gender which found to have an increased risk of dementia. There was significant correlation of the above parameters with MoCA scales and ACE, previous literature has shown that low education is a risk factor for cognitive impairment in older age. Several mechanisms have been proposed to explain the relationship between low education and cognitive decline in several studies earlier; education may be a marker of other factors related to cognition. ${ }^{22,23}$

Our findings emphasized that the incidence of dementia in the subjects decreased proportionately as the level of education among society was increased. Mortimer at al proposed a relationship between years of formal education and risk for dementia. ${ }^{24}$ Saldanha Dat el and co-workers also revealed the positive correlation between low levels of education with an increased incidence of dementia. ${ }^{25,26}$

The sensitivity of MoCA, ACE, and EASI score was found to be extremely high which is indicative of the fact that these tools have a good capability to detect most patients with a morbid condition, i.e., it is sensitive enough to predict probable cases of dementia. 
Larner, et al 2012 studies with MoCA showed a sensitivity $0.93(0.77-0.99)$ and a specificity of 0.60 (0.49-0.70) while Cummings-Vaughn et al, 2014 studies with MoCA showed a sensitivity $0.81(0.68$ $0.90)$ and a specificity of $0.75(0.64-0.84) .^{27,28}$ The difference in sensitivity and specificity of various studies is because different studies use different cut off. EASI had sensitivities $62.5 \%$, with specificities of $89.7 \%$, in another study by Raina et al. ${ }^{29}$ An advantage of the EASI was that it could also be administered to informants of subjects who were cognitively untestable. In this largely illiterate community, with a low prevalence of dementia, the combination of cognitive tests and a functional ability questionnaire had substantial value for population screening.

Diagnostic sensitivity improves with lower cutoff values but with a corresponding decrease in specificity. High sensitivity corresponds to high negative predictive value and is the ideal to rule out dementia. There was considerable variation on the definitions of cutoff thresholds among the individual studies. ${ }^{30}$ More patients would be required to further confine the sensitivity and specificity. Results may have been affected by test order, sample size and education level.

Table 1: Socio-demographic profile of patients

\begin{tabular}{|l|l|l|}
\hline \multicolumn{1}{|c|}{ Parameters } & N & $(\%)$ \\
\hline Age Group (yrs) & 24 & 10.3 \\
\hline $20-49$ & 41 & 17.6 \\
\hline $50-59$ & 84 & 36.1 \\
\hline $60-69$ &
\end{tabular}

\begin{tabular}{|l|c|c|}
\hline $70-79$ & 69 & 29.6 \\
\hline $80+$ & 15 & 6.4 \\
\hline Occupation \\
\hline Business & 63 & 27.0 \\
\hline Farmer & 17 & 7.3 \\
\hline House Wife & 42 & 18.0 \\
\hline Service & 109 & 46.8 \\
\hline Student & 2 & 0.9 \\
\hline Education & 25 & 10.7 \\
\hline Illiterate & 47 & 20.2 \\
\hline Primary & 34 & 14.6 \\
\hline SSC & 30 & 12.9 \\
\hline HSC & 61 & 26.2 \\
\hline Degree & 36 & 15.5 \\
\hline Post Graduate \& above & 119 & 50.9 \\
Addiction
\end{tabular}

$\mathrm{N}=$ Number of patients; $\mathrm{SSC}=$ Secondary School Certificate; and $\mathrm{HSC}=$ Higher Secondary Certificate

Table 2: Cognitive impairment score of various assessment

\begin{tabular}{|l|c|c|c|c|}
\hline Scores & N & Min & Max & Mean \pm SD \\
\hline MoCA & 233 & 4 & 27 & $14.64 \pm 5.51$ \\
\hline EASI & 233 & 0 & 12 & $7.17 \pm 2.35$ \\
\hline HIS & 233 & 2 & 10 & $4.20 \pm 2.39$ \\
\hline ACE & 233 & 11 & 77 & $46.64 \pm 12.45$ \\
\hline
\end{tabular}

$\mathrm{MoCA}=$ Montreal Cognitive Assessment; EASI= Everyday Abilities Scale for India; HIS= Hachinski Ischemic Score, and $\mathrm{ACE}=$ Addenbrooke's Cognitive Examination. The Data has been arranged as Mean \pm SD.

Table 3: Pearson's correlations matrix of age for selective parameters

\begin{tabular}{|l|c|c|c|c|c|}
\hline \multicolumn{7}{|c|}{$\begin{array}{c}\text { Pearson Correlation Coefficients, } \mathbf{N}=\mathbf{2 3 3} \\
\text { Prob }>|\mathbf{r}|\end{array}$ under H0: Rho=0 } \\
\hline & Age & Moca & Easi & His & Ace \\
\hline Age & 1.00000 & -0.24388 & 0.11689 & 0.03424 & -0.23287 \\
& & $0.0002^{*}$ & 0.0749 & 0.6031 & $0.0003^{*}$ \\
\hline Moca & -0.24388 & 1.00000 & -0.64774 & -0.02377 & 0.87282 \\
& $0.0002^{*}$ & & $<.0001^{*}$ & 0.7181 & $<.0001^{*}$ \\
\hline Easi & 0.11689 & -0.64774 & 1.00000 & 0.07453 & -0.64426 \\
& 0.0749 & $<.0001^{*}$ & & 0.2572 & $<.0001^{*}$ \\
\hline His & 0.03424 & -0.02377 & 0.07453 & 1.00000 & -0.14057 \\
& 0.6031 & 0.7181 & 0.2572 & & 0.0320 \\
\hline Ace & -0.23287 & 0.87282 & -0.64426 & -0.14057 & 1.00000 \\
& $0.0003^{*}$ & $<.0001^{*}$ & $<.0001^{*}$ & 0.0320 & \\
\hline
\end{tabular}

All the data were expressed as Pearson's correlations coefficient $(r)$ and $p$ value. . MoCA $=$ Montreal Cognitive Assessment; EASI= Everyday Abilities Scale for India; HIS= Hachinski Ischemic Score, and ACE= Addenbrooke's Cognitive Examination. If $(\mathrm{p} \leq 0.05)$ then it was considered as statistically significant

Table 4: Correlations Matrix of sex for selective parameters

\begin{tabular}{|l|c|c|}
\hline & Male- $(\mathbf{N} \pm$ SD $)$ & Female $-(\mathbf{N} \pm$ SD $)$ \\
\hline MoCA & $15.55 \pm 5.38$ & $10.75 \pm 4.28$ \\
\hline EASI & $7.00 \pm 2.30$ & $7.91 \pm 2.43$ \\
\hline HIS & $4.31 \pm 2.45$ & $3.73 \pm 2.05$ \\
\hline ACE & $48.56 \pm 12.11$ & $38.41 \pm 10.48$ \\
\hline Total & 189 & 44 \\
\hline
\end{tabular}


All the data were expressed in Mean \pm SD. MoCA= Montreal Cognitive Assessment; EASI= Everyday Abilities Scale for India; $\mathrm{HIS}=$ Hachinski Ischemic Score, and ACE= Addenbrooke's Cognitive Examination.

Table 5: Correlations matrix of education for selective parameters

\begin{tabular}{|l|c|c|c|c|c|c|c|}
\hline & Illiterate & Primary & SSC & HSC & Degree & PG & P value \\
\hline MoCA & $10.00 \pm 3.86$ & $11.77 \pm 3.92$ & $14.32 \pm 5.00$ & $16.90 \pm 4.04$ & $16.51 \pm 5.66$ & $16.89 \pm 6.05$ & $<0.001^{*}$ \\
\hline EASI & $8.60 \pm 1.98$ & $7.53 \pm 2.51$ & $7.06 \pm 1.76$ & $6.90 \pm 1.86$ & $6.25 \pm 2.23$ & $7.61 \pm 2.82$ & $<0.001^{*}$ \\
\hline HIS & $4.28 \pm 2.53$ & $4.06 \pm 2.29$ & $4.26 \pm 2.48$ & $4.17 \pm 2.39$ & $4.56 \pm 2.54$ & $3.67 \pm 2.11$ & 0.672 \\
\hline ACE & $36.72 \pm 8.40$ & $41.77 \pm 9.64$ & $47.15 \pm 9.96$ & $50.90 \pm 10.87$ & $49.69 \pm 13.21$ & $50.72 \pm 14.68$ & $<0.001^{*}$ \\
\hline
\end{tabular}

All the data were expressed in Mean \pm SD. MoCA= Montreal Cognitive Assessment; EASI= Everyday Abilities Scale for India; $\mathrm{HIS}=$ Hachinski Ischemic Score, and ACE= Addenbrooke's Cognitive Examination. If $(\mathrm{p} \leq 0.05)$ then it was considered as statistically significant.

Table 6: MoCA score, EASI score and HIS score with: sensitivity, specificity, PPV (positive predictive value), NPV (negative predictive value)

\begin{tabular}{|l|c|c|c|c|}
\hline \multicolumn{1}{|c|}{ Test } & $\begin{array}{c}\text { Sensitivity } \\
\text { (95\% CI) }\end{array}$ & $\begin{array}{c}\text { Specificity } \\
(\mathbf{9 5 \%} \text { CI) }\end{array}$ & $\begin{array}{c}\text { PPV } \\
(\mathbf{9 5 \%} \text { CI })\end{array}$ & $\begin{array}{c}\text { NPV } \\
(\mathbf{9 5 \%} \text { CI) }\end{array}$ \\
\hline MoCA & $72.69 \%$ & $50.00 \%$ & $98.21 \%$ & $4.62 \%$ \\
\hline ACE & $72.29 \%$ & $100 \%$ & $100 \%$ & 0.00 \\
\hline EASI & $71.74 \%$ & $0.00 \%$ & $98.21 \%$ & $0 \%$ \\
\hline
\end{tabular}

$\mathrm{MoCA}=$ Montreal Cognitive Assessment; EASI= Everyday Abilities Scale for India, and ACE= Addenbrooke's Cognitive Examination.

\section{Conclusion}

Currently, available short cognitive scales allow only the identification of cognitive shortfalls in patients. Recognition of cognitive shortfalls is necessary for the management of patients with cognitive problems. Doctors using short tests are examining the patient not performing a diagnostic procedure. Over-reliance on very short, poorly validated tests may lead to mass over or under diagnosis of dementia.

The present study provides information regarding the utility of the MoCA, ACE and EASI as an efficient diagnostic tool, in quick screening of cognitive impairments. These instruments appear to be reliable tools for the screening of dementia in limited setting of study. However, this finding needs to be validated for larger patient population spread across India.

\section{Acknowledgment: None}

\section{References}

1. Ferri CP, Prince M, Brayne C, Brodaty H, Fratiglioni L, et al. Global prevalence of dementia: a Delphi consensus study. Lancet 2005;366:2112-17.

2. Shaji KS, Jotheeswaran AT, Girish N, Srikala B, Dias A, et al. Alzheimer's \& Related Disorders Society of India, The Dementia India Report: prevalence, impact, costs and services for Dementia: Executive Summary. (Eds), ARDSI, New Delhi

3. NICE. (2006) Dementia: supporting people with dementia and their careers in health and social care. Clinical guideline 42. London: National Institute for Health and Clinical Excellence.

4. National Audit Office. Improving Dementia Services in England-An Interim Report. London, UK: National AuditOffice, 2010. http:// www.nao.org.uk/publications/0910/improving_dementia_ services. aspx (accessed on11 October 2010).
5. Linda Nazarko. Cognitive assessment: a guide for community nurses. Br J Community Nurs 18(11):550-53.

6. David R. Gifford, MD, MPH; and Jeffrey L. Cummings, MD. Evaluating dementia screening tests methodological standards to rate their performance. Neurol 1999;52:22427.

7. Qui C, Kivipelto M, von Strauss E. Epidemiology of Alzheimer disease: Occurrence, determinants, and strategies toward intervention. Dialogues Clin Neurosci 2009;11(2):111-28.

8. www.lhsc.on.ca/ programs/ msclinic/ define/ c.htm last accessed 2017 Nov 07.

9. Snowdon DA, Greiner LH, Mortimer JA, Riley KP, Greiner PA, Markesbery WR. Brain infarction and the clinical expression of Alzheimer disease. The Nun Study. JAMA 1997;277:813-17.

10. Brucki SMD. Illiteracy and dementia. Dement Neuropsychol 2010;4(3):153-57.

11. Borson S, Scanlan JM, Watanabe J, Tu SP, Lessig M. Improving identification of cognitive impairment in primary care. Int J Geriatr Psychiatry 2006;21(4):349-55.

12. Nasreddine, Ziad S., Phillips, Natalie A., Bédirian, Valérie. et al. "The Montreal Cognitive Assessment, MoCA: a brief screening tool for mild cognitive impairment". J Am Geriatrics Soc 2005;53(4):695-99.

13. Mioshi, E., Dawson, K., Mitchell, J., Arnold, R., \& Hodges, J. R. The Addenbrooke's Cognitive Examination Revised (ACE-R): a brief cognitive test battery for dementia screening. Int J Geriatric Psychiatry 2006;21(11):1078-85.

14. Hsieh, S., Schubert, S., Hoon, C., Mioshi, E., \& Hodges, J. R. Validation of the Addenbrooke's Cognitive Examination III in fronto-temporal dementia and Alzheimer's disease. Dementia Geriatric Cognitive Disorders 2013;36(3-4):242-50.

15. Shuba N, Karan. Assessment of the Cognitive Status in Diabetes Mellitus. J Clin Diagnostic Res 2012;6(10):1658-62.

16. Andrew J Larner. Cognitive screening instruments for the diagnosis of mild cognitive impairment. Progress Neurol Psychiatry 2016;20(2):21-6. 
17. Uvlund K, Kreiner S, Schultz-Larsen K. Functional ability scales for elderly: A validation study. Eur J Public Health 1996;6:35-42.

18. Pantoni L1, Inzitari D. Hachinski's ischemic score and the diagnosis of vascular dementia: a review. Ital J Neurol Sci 1993;14(7):539-46.

19. Monsch AU, Foldi NS, Ermini-Funfschilling DE, Berres M, Taylor K I, et al. Improving the diagnostic accuracy of the mini mental state examination. Acta Neurologica Scandinavica 1995;92:45-50.

20. Rajesh Pandav, Gerda Fillenbaum, Graham Ratcliff, Hiroko Dodge, and Mary Ganguli. Sensitivity and Specificity of Cognitive and Functional Screening Instruments for Dementia: The Indo-U.S. Dementia Epidemiology Study. J Am Geriatr Soc 2002;50(3):55461.

21. Gowri K. Iyer, Suvarna Alladi. Dementia in developing countries. Does education play the same role in India as in the West? Dement Neuropsychol 2014;8(2):132-40.

22. Zhang ZX, Zahner GE, Román GC, et al. Dementia subtypes in China: prevalence in Beijing, Xian, Shanghai, and Chengdu. Arch Neurol 2005;62:447-54.

23. Barnes DE, Yaffe K. The projected effect of risk factor reduction on Alzheimer's disease prevalence. Lancet Neurol 2011;10(9):819-28.

24. Mortimer JA, Snowden DA, Markesbery WR. Head circumference, education and risk of dementia: findings from the Nun Study. J Clin Exp Neuropsychol 2003;25:671-79.
25. Saldanha D, Mani MR, Srivastava K, Goyal S, Bhattacharya D. "An Epidemiological Study of Dementia under the Aegis of Mental Health mental health program, Maharashtra, Pune chapter. Indian J Psychiatry 2010;52(2):131-39.

26. Gaidhane S, Gaidhane AM, Zahiruddin QS, Khatib N. Essential hypertension and cognitive function in elderly. Global J Med Public Health (2014);3:1-12.

27. Larner AJ. Screening utility of the Montreal Cognitive Assessment (MoCA): in place of - or as well as - the MMSE? Int Psycho Geriatr 2012;24(3):391-96.

28. Cummings-Vaughn LA, Chavakula NN, Malmstrom TK, Tumosa N, Morley JE, Cruz-Oliver DM. Veterans Affairs Saint Louis University Mental Status Examination compared with the Montreal Cognitive Assessment and the Short Test of Mental Status. J Am Geriatr Soc 2014;62(7):1341-46.

29. Raina SK, Chander V, Raina S, Kumar D. Feasibility of using everyday abilities scale of India as alternative to mental state examination as a screen in two-phase survey estimating the prevalence of dementia in largely illiterate Indian population. Indian J Psychiatry 2016;58(4):45961.

30. Kelvin K. F. Tsoi; Joyce Y. C. Chan, MPH; Hoyee W. Hirai; Samuel Y. S. Wong; Timothy C. Y. Kwok. Cognitive Tests to Detect Dementia: A Systematic Review and Meta-analysis. JAMA Internal Med 2015;175(9):1450-58. 